\title{
Hypermethylation involved in DNA profiles of lung cancer specific tumour suppressor genes and epigenetic modification caused by an ultra-highly diluted homeopathic drug, Condurango 30C, in vitro and in vivo
}

\author{
Sourav Sikdar, Anisur Rahman Khuda Bukhsh
}

Cytogenetics and Molecular Biology Laboratory, University of Kalyani, West Bengal, India

\begin{abstract}
Background and objectives: DNA hyper-methylation is an important aspect involved in carcinogenesis and cancer progression, which affects mainly cpg island of DNA and causes inactivation of tumour suppressor genes. Therefore DNA hypermethylation status of the genomic DNA in both the transformed cancerous cell lines and in carcinogen-induced lung cancer was ascertained by analysis of expressions of certain major lung cancer specific tumour suppressor genes. The other objective was to examine if ultra highly diluted homeopathic drug, Condurango 30C, had ability to modulate DNA methylation. Methods: DNA methylation activity, if any, has been ascertained in H460-NSCLC cells in vitro and in bap-induced lung cancer of rats in vivo, in respect of tumour suppressor genes like p15, p16, p18 and p53 by using PCR-SSCP analyses. The ability of modulation of DNA methylation, if any, by Condurango 30C was also verified against placebo control in a blinded manner. Results: Condurango 30C-treated DNA showed significant decrease in band-intensity of $\mathrm{p} 15$ and $\mathrm{p} 53$ genes especially in methylated condition, in vitro, at the $\mathrm{IC}_{50}$ dose $(2.43 \mu \mathrm{l} / 100 \mu \mathrm{l})$. SSCP analysis of p15 and p53 genes in Condurango 30C-treated DNA also supported ability of Condurango 30C to modulate methylation state, in vitro. Inhibition of p15 hypermethylation was observed after post cancer treatment of rat with Condurango 30C. SSCP results gave a better indication of differences in band-position and single strand separation of $\mathrm{p} 15$ and p53 in Condurango 30C treated samples. Conclusion: Condurango $30 \mathrm{C}$ could trigger epigenetic modification in lung cancer via modulation of DNA hypermethylation but placebos could not.
\end{abstract}

Keywords: DNA methylation, CpG islands, epigenetic modification, Condurango 30C

\section{(c)) EY-NC-ND Licensed to GIRI}

Support: The study partial funding from a grant from Boiron Laboratories, Lyon, France

Conflict of interest: None to declare

Received: April 10, 2014; Revised: May 10 th 2014; Published: June 30 2014.

Correspondence author: Anisur Rahman Khuda-Bukhsh; prof arkb@yahoo.com.in; khudabukhsh 48@rediffmail.com

How to cite this article: Surname: Khuda-Bukhsh AR and Sikdar S. Hypermethylation involved in DNA profiles of lung cancer specific tumour suppressor genes and epigenetic modification caused by an ultra-highly diluted homeopathic drug, Condurango 30C, in vitro and in vivo. Int J High Dilution Res [online]. 2014 [cited YYYY Month dd]; 13(47):99-99. Proceedings of the XXVIII GIRI Symposium; 2014 Jun 20-22; Sighisoara (Romania). GIRI; 2014; Available from: http://www.feg.unesp.br/ ojs/index.php/ijhdr/article/view/721/700 